\title{
Necessary And Sufficient Condition of Separability of Any System
}

\author{
Ping-Xing Chen and Lin-Mei Liang \\ Laboratory of Quantum Communication and Quantum Computation, \\ University of Science and Technology of \\ China, Hefei, 230026, P. R. China \\ and \\ * Department of Applied Physics, National University of \\ Defense Technology, \\ Changsha, 410073, \\ P. R. China \\ Cheng-Zu Li \\ Department of Applied Physics, \\ National University of Defense Technology, \\ Changsha, 410073, P. R. China \\ Ming-Qiu Huang \\ CCAST (World Laboratory) P.O. Box 8730, Beijing, 100080, China \\ and Department of Applied Physics, \\ National University of Defense Technology, \\ Changsha, 410073, P. R. China
}

(November 14, 2018)

\begin{abstract}
The necessary and sufficient condition of separability of a mixed state of any systems is presented, which is practical in judging the separability of a mixed state. This paper also presents a method of finding the disentangled decomposition of a separable mixed state.
\end{abstract}

PACS number(s): 03.67.-a, 03.65.Bz

${ }^{*}$ Corresponding address 
Entanglenment is an important element both in quantum theory [1] and in quantum information [2 5]. A mixed state $\rho$, which acts on Hilbert space $H_{1} \otimes H_{2}$, has no entanglement or is separable iff $\rho$ can be written as the following form [6]

$$
\rho=\sum_{i} p_{i} \rho_{i}^{1} \otimes \rho_{i}^{2}
$$

where $\rho_{i}^{1}$ and $\rho_{i}^{2}$ are states of Hilbert space $H_{1}$ and $H_{2}$, respectively, and $p_{i} \geq 0, \sum p_{i}=1$. A separable system satisfies the Bell inequality, but the converse is not necessarily true [7]. Peres has shown in Ref. [8] that a necessary condition (NC) for separability of a state $\rho$ is the positivity of its partial transformation $\rho^{T}$. Here $\rho^{T}$ is defined as

$$
\rho_{m \mu, n \nu}^{T_{1}} \equiv \rho_{n \mu, m v}, \quad \rho_{m \mu, n \nu}^{T_{2}} \equiv \rho_{m \nu, n \mu}
$$

It has been shown that for $2 \times 2$ systems (the dimensions of $H_{1}$ and $H_{2}$ are 2 and 2 , respectively) and $2 \times 3$ systems, the condition (2) is also a sufficient one [9]. A necessary and sufficient condition(NSC) for $m \times n$ systems with the aid of the concept of a map has been presented by Horodecki et.al in Ref. [9], but it is difficult to judge whether a state is separable or not in practice with this method. W.Dür et.al [10] show a NSC of a certain family of mixed states. In this paper, we shall present a NSC of separability of $\rho$ for any system. Our main idea is based on the idea proposed by Wootters in Ref. 11,12]. Compared with Horodecki et.al's work, the NSC presented in this paper is more explicitly algorithmic, so it is practical in operation. The organization of this paper is as follows. First, we collect some facts related to our result. Second, the NSC of any mixed state is presented. Meanwhile, how to get the disentangled decomposition of a separable state is also presented. Finally, we present an example of a bound entangled state as an illustration. From this example, one can know how to judge whether a mixed state is separable or not.

In order to get the results, we collect the facts as follows:

1.Any mixed state $\rho$ of $m \times n$ systems which has $l$ nonzero eigenvalues can be decomposed into $k$ pure states. And the number of possible decompositions of $\rho$ is infinite. These decompositions can be obtained by some transformation $u_{k \times l}$ whose columns are orthonormal vectors ( $k$ is greater than or equal to $l$ ) [11]. For example,

$$
\rho=\sum_{i=1}^{l}\left|x_{i}\right\rangle\left\langle x_{i}\left|=\sum_{i=1}^{k}\right| z_{i}\right\rangle\left\langle z_{i}\right|,
$$

where $\left|x_{i}\right\rangle$, unnormalized, is a complete set of orthogonal eigenvectors corresponding to the nonzero eigenvalues of $\rho$, and $\left\langle x_{i} \mid x_{i}\right\rangle$ is equal to the ith nonzero eigenvalue. Then the decomposition $\left|z_{i}\right\rangle$ of $\rho$ can be given by

$$
\left|z_{i}\right\rangle=\sum_{j=1}^{l} u_{i j}\left|x_{j}\right\rangle, \quad i=1,2, \cdots, k
$$


where the vectors $\left|z_{i}\right\rangle$ are not necessarily orthogonal.

2. A pure state $|\Psi\rangle$ of an $m \times n$ systems is denoted as

$$
|\Psi\rangle=A_{11}|11\rangle+A_{12}|12\rangle+\cdots+A_{1 n}|1 n\rangle+\cdots+A_{m 1}|m 1\rangle+\cdots+A_{m n}|m n\rangle,
$$

where $|11\rangle, \cdots|1 n\rangle, \cdots|m 1\rangle, \cdots|m n\rangle$ are the standard basis vectors of the $m \times n$ system. We introduce the "vector" $\overrightarrow{A_{i}}$

$$
\overrightarrow{A_{i}}=\left(A_{i 1}, A_{i 2}, \cdots A_{i n}\right) . \quad i=1,2, \cdots, m
$$

It is not difficult to find that the pure state $|\Psi\rangle$ is separable if and only if all the "vectors" $\overrightarrow{A_{i}}$ are parallel with another, which is denoted as

$$
\overrightarrow{A_{1}}\left\|\overrightarrow{A_{2}}\right\| \cdots \| \overrightarrow{A_{m}}
$$

Note that the relation(7) is true if and only if the following is true

$$
\left\{\begin{array}{c}
\left(A_{11}, A_{12}\right)\left\|\left(A_{21}, A_{22}\right)\right\| \cdots \|\left(A_{m 1}, A_{m 2}\right) \\
\left(A_{11}, A_{13}\right)\left\|\left(A_{21}, A_{23}\right)\right\| \cdots \|\left(A_{m 1}, A_{m 3}\right) \\
\vdots \\
\left(A_{11}, A_{1 n}\right)\left\|\left(A_{21}, A_{2 n}\right)\right\| \cdots \|\left(A_{m 1}, A_{m n}\right)
\end{array} .\right.
$$

That is to say there exist $(m-1)(n-1)$ parallel pairs. For example, having parallel pairs $\left(A_{11}, A_{12}\right)\left\|\left(A_{21}, A_{22}\right),\left(A_{11}, A_{12}\right)\right\|\left(A_{31}, A_{32}\right)$ means that $A_{11} / A_{21}=A_{12} / A_{22}, A_{11} / A_{31}=$ $A_{12} / A_{32}$, respectively.

3. We define $\left|\widetilde{\Psi}^{r}\right\rangle$ and $c_{r}$ with respect to each parallel pair as follows:

$$
\left|\widetilde{\Psi}^{r}\right\rangle=B^{r}\left|\Psi^{*}\right\rangle, c_{r}=\left\langle\Psi \mid \widetilde{\Psi}^{r}\right\rangle, \quad r=1,2, \cdots,(m-1)(n-1)
$$

where $B^{r}$ is a $m n \times m n$ symmetric transformation matrix in the standard basis and there are only 4 nonzero elements of $B^{r}$. For example, for $\left(A_{11}, A_{12}\right) \|\left(A_{21}, A_{22}\right),\left[B^{1}\right]_{1, n+2}=$ $\left[B^{1}\right]_{n+2,1}=-1,\left[B^{1}\right]_{2, n+1}=\left[B^{1}\right]_{n+1,2}=1$, the other elements of $\left[B^{1}\right]$ are all zero. It is obvious that $\left|c_{1}\right|=0$ if and only if $\left(A_{11}, A_{12}\right) \|\left(A_{21}, A_{22}\right)$. Similarly, each parallel pair in the relation(8) can be expressed by its $\left|c_{r}\right|$ being equal to zero and this is also the reason we choose the elements of the matrix $B^{r}$. For $4 \times 4$ systems, there exists nine transformation matrices as follows:

$$
\begin{aligned}
& \left(A_{11}, A_{12}\right) \|\left(A_{21}, A_{22}\right) \rightarrow\left[B^{1}\right]_{1,6}=-1,\left[B^{1}\right]_{2,5}=1,\left[B^{1}\right]_{5,2}=1,\left[B^{1}\right]_{6,1}=-1, \\
& \left(A_{11}, A_{12}\right) \|\left(A_{31}, A_{32}\right) \rightarrow\left[B^{2}\right]_{1,10}=-1,\left[B^{2}\right]_{2,9}=1,\left[B^{2}\right]_{9,2}=1,\left[B^{2}\right]_{10,1}=-1, \\
& \left(A_{11}, A_{12}\right) \|\left(A_{41}, A_{42}\right) \rightarrow\left[B^{3}\right]_{1,14}=-1,\left[B^{3}\right]_{2,13}=1,\left[B^{3}\right]_{13,2}=1,\left[B^{3}\right]_{14,1}=-1, \\
& \left(A_{11}, A_{13}\right) \|\left(A_{21}, A_{23}\right) \rightarrow\left[B^{4}\right]_{1,7}=-1,\left[B^{4}\right]_{3,5}=1,\left[B^{4}\right]_{5,3}=1,\left[B^{4}\right]_{7,1}=-1, \\
& \left(A_{11}, A_{13}\right) \|\left(A_{31}, A_{33}\right) \rightarrow\left[B^{5}\right]_{1,11}=-1,\left[B^{5}\right]_{3,9}=1,\left[B^{5}\right]_{9,3}=1,\left[B^{5}\right]_{11,1}=-1,
\end{aligned}
$$




$$
\begin{aligned}
& \left(A_{11}, A_{13}\right) \|\left(A_{41}, A_{43}\right) \rightarrow\left[B^{6}\right]_{1,15}=-1,\left[B^{6}\right]_{3,13}=1,\left[B^{6}\right]_{13,3}=1,\left[B^{6}\right]_{15,1}=-1, \\
& \left(A_{11}, A_{14}\right) \|\left(A_{21}, A_{24}\right) \rightarrow\left[B^{7}\right]_{1,8}=-1,\left[B^{7}\right]_{4,5}=1,\left[B^{7}\right]_{5,4}=1,\left[B^{7}\right]_{8,1}=-1, \\
& \left(A_{11}, A_{14}\right) \|\left(A_{31}, A_{34}\right) \rightarrow\left[B^{8}\right]_{1,12}=-1,\left[B^{8}\right]_{4,9}=1,\left[B^{8}\right]_{9,4}=1,\left[B^{8}\right]_{12,1}=-1, \\
& \left(A_{11}, A_{14}\right) \|\left(A_{41}, A_{44}\right) \rightarrow\left[B^{9}\right]_{1,16}=-1,\left[B^{9}\right]_{4,13}=1,\left[B^{9}\right]_{13,4}=1,\left[B^{9}\right]_{16,1}=-1,
\end{aligned}
$$

the other elements of $\left[B^{r}\right](r=1, \cdots, 9)$ are all zero. Similarly, for higher dimension systems, we choose $B^{r}$ of each parallel pairs so that each $c_{r}$ is zero.

4. From the above facts, one can get that any state $\rho$ is separable iff there is a pure state decomposition of $\rho, \rho=\sum_{i}\left|z_{i}\right\rangle\left\langle z_{i}\right|$ with each $\left|z_{i}\right\rangle$ being separable, i.e., each pair in Eq.(8) is parallel for all $\left|z_{i}\right\rangle$.

In the following, we will discuss the NSC of separability of $m \times n$ systems. Firstly, we will prove the following lemma.

Lemma. For any density matrix $\rho$ of $m \times n$ systems in Eq.(3), the statement $\left(A_{11}, A_{12}\right) \|$ $\left(A_{21}, A_{22}\right)$ is true for each pure state $\left|z_{i}\right\rangle$ in Eq.(田) if and only if

$$
a^{1}=\lambda_{1}-\sum_{i=2}^{l^{\prime}} \lambda_{i} \leq 0
$$

where $l^{\prime} \leq l\left(l, l^{\prime}\right.$ is the number of eigenvalues of $\rho, \tau^{1}\left(\tau^{1}\right)^{*}$ respectively. $)$ and $\lambda_{i} \mathrm{~s}$, in decreasing order, are the square roots of eigenvalues of the matrix $\tau^{1}\left(\tau^{1}\right)^{*}$, where $\tau_{i j}^{1}=$ $\left\langle x_{i} \mid \widetilde{x}_{j}^{1}\right\rangle$ and $\left|x_{i}\right\rangle$ is defined in Eq.(3) and $\left|\widetilde{x}_{j}^{1}\right\rangle$ in Eq.(9)).

Proof: Obviously $\tau^{1}$ is a symmetric matrix. For the symmetric matrix $\left[\tau_{i j}^{1}\right]$, one can find a $l \times l$ unitary matrix $\left[\nu_{i j}\right]$ which can diagonalize the $\left[\tau_{i j}^{1}\right]$ in the following way

$$
\left\langle y_{i} \mid \widetilde{y}_{j}^{1}\right\rangle=\left[v \tau^{1} v^{T}\right]_{i j}=\lambda_{i} \delta_{i j}
$$

where $\left|y_{i}\right\rangle=\sum_{j} \nu_{i j}^{*}\left|x_{j}\right\rangle, \nu_{i j}^{*}$ is the complex conjugation of $\nu_{i j}$, and $\lambda_{i}$ s are the square roots of eigenvalues of matrix $\tau^{1}\left(\tau^{1}\right)^{*}$ [11]. Though $\tau^{1}$ is dependent on the choice of eigenvectors $\left|x_{i}\right\rangle$ of $\rho, \lambda_{i}$ is not. Every decomposition of $\rho, \rho=\sum_{i}\left|z_{i}\right\rangle\left\langle z_{i}\right|$, can be obtained by $\left|z_{i}\right\rangle=\sum_{j} \mu_{i j}^{*}\left|y_{j}\right\rangle$. Here

$$
\mu^{*}=\left[\begin{array}{rrrr}
a_{1,1} e^{i \theta_{1,1}} & a_{1,2} e^{i \theta_{1,2}} & \ldots & a_{1, l} e^{i \theta_{1, l}} \\
a_{2,1} e^{i \theta_{2,1}} & a_{2,2} e^{i \theta_{2,2}} & \ldots & a_{2, l} e^{i \theta_{2, l}} \\
& \vdots & & \\
a_{k, 1} e^{i \theta_{k, 1}} & a_{k, 2} e^{i \theta_{k, 2}} & \ldots & a_{k, l} e^{i \theta_{k, l}}
\end{array}\right]
$$

whose columns are orthonormal vectors and there is no loss of generality in taking each $a_{i j}$ to be real and nonnegative. If $l^{\prime}<l$, one can add $\left(l-l^{\prime}\right)$ dummy states $\left|y_{l^{\prime}+1}\right\rangle,\left|y_{l^{\prime}+2}\right\rangle, \cdots,\left|y_{l}\right\rangle$ being equal to zero vectors. Thus it can be obtained that $\left|z_{i}\right\rangle=$ $\sum_{j} u_{i j}^{1}\left|x_{j}\right\rangle$, where $u^{1}=\mu^{*} \nu^{*}$ (there may exist many transformation matrices which can realize this kind of decomposition. The set which contains all these transformation matrices is denoted by $\left.U_{k \times l}^{1}\right)$. If $\left(A_{11}, A_{12}\right) \|\left(A_{21}, A_{22}\right)$ for each $\left|z_{i}\right\rangle$, from fact 3 , one has 


$$
\left\langle z_{i} \mid \widetilde{z}_{i}^{1}\right\rangle=0
$$

where $\left|\widetilde{z}_{i}^{1}\right\rangle=B^{1}\left|z_{i}^{*}\right\rangle$; then one has

$$
\begin{aligned}
a_{i 1}^{4} \lambda_{1}^{2} & =\left(\sum_{j=2}^{l^{\prime}} a_{i j}^{2} \cos 2 \theta_{i j} \lambda_{j}\right)^{2}+\left(\sum_{j=2}^{l^{\prime}} a_{i j}^{2} \sin 2 \theta_{i j} \lambda_{j}\right)^{2} \\
& \leq\left(\sum_{j=2}^{l^{\prime}} a_{i j}^{2} \lambda_{j}\right)^{2} .
\end{aligned}
$$

Because of the positivity of $\lambda_{i}$ and $a_{i j}$, one can get

$$
a_{i 1}^{2} \lambda_{1} \leq \sum_{j=2}^{l^{\prime}} a_{i j}^{2} \lambda_{j},
$$

and then, $\lambda_{1}=\sum_{i=1}^{k} a_{i 1}^{2} \lambda_{1} \leq \sum_{i=1}^{k} \sum_{j=2}^{l^{\prime}} a_{i j}^{2} \lambda_{j}=\sum_{j=2}^{l^{\prime}} \lambda_{j}$, i.e.

$$
a^{1}=\lambda_{1}-\sum_{i=2}^{l^{\prime}} \lambda_{i} \leq 0 \text {. }
$$

Here we have used Eq.(15) and $\sum_{i=1}^{k} a_{i 1}^{2}=1$.

Conversely, if $\lambda_{1} \leq \sum_{i=2}^{l^{\prime}} \lambda_{i}$, one can find a kind of decomposition of $\rho, \rho=\sum_{i}\left|z_{i}\right\rangle\left\langle z_{i}\right|$ [see Appendix], where $\left\langle z_{i} \mid \widetilde{z}_{i}^{1}\right\rangle=0$, so that $\left(A_{11}, A_{12}\right) \|\left(A_{21}, A_{22}\right)$. Therefore the lemma is proved.

Similarly, from the proof of the Lemma one knows that for an $m \times n$ system the NSC for each of the $(m-1)(n-1)$ pairs being parallel is $a^{r} \leq 0(r=1,2, \cdots,(m-$ $1)(n-1))$. If each $a^{r} \leq 0$, there exist $(m-1)(n-1)$ sets of transformation matrices, $U_{k \times l}^{1}, U_{k \times l}^{2}, \cdots, U_{k \times l}^{(m-1)(n-1)}$, each of which yields decompositions of $\rho$ with the existence of the corresponding parallel pair. Thus we get the main result in this paper:

Theorem. A NSC of separability of states of an $m \times n$ system is that the corresponding $a^{r} \leq 0 \quad(r=1, \cdots,(m-1)(n-1))$ and the intersection $U_{k \times l}$ of the $(m-1)(n-1)$ sets of transformations is not empty.

Proof: The sufficient condition is obvious. The task left is to prove the necessary condition. If $\rho$ is separable, there must exist a transformation matrix $u$ which can transform the decomposition of $\rho, \rho=\sum_{i=1}^{l}\left|x_{i}\right\rangle\left\langle x_{i}\right|$ into $\rho=\sum_{i}\left|z_{i}\right\rangle\left\langle z_{i}\right|$ with the existence of $(n-$ 1) $(m-1)$ parallel pairs in each pure state $\left|z_{i}\right\rangle$. From the Lemma, one can get each $a^{r} \leq 0$ in each $\left|z_{i}\right\rangle$, and the matrix $u$ belongs to the intersection $U_{k \times l}$; i.e. $U_{k \times l}$ is not empty. Thus the theorem is proved.

According to the above theorem, one may take the following steps (i)-(iv) to judge whether a state $\rho$ of an $m \times n$ quantum system is separable or not.

i). Calculate the nonzero eigenvalues and eigenvectors of $\rho$ (see Eq.(3)). 
ii). Calculate $(m-1)(n-1)$ matrices $\tau^{r}\left(\tau^{r}\right)^{*}$ according to $B^{r}(r=1,2, \cdots,(m-1)(n-1))$ and obtain the square roots of the eigenvalues of $\tau^{r}\left(\tau^{r}\right)^{*}$ (see Eq.(11)).

iii). Judge whether $a^{r}(r=1,2, \cdots,(m-1)(n-1))$ is greater than zero or not (see Eq.(10)). If all $a^{r} \leq 0$, then go to step (iv), otherwise $\rho$ is inseparable.

iv). Judge whether the intersection of $(m-1)(n-1)$ sets of transformation $U_{k \times l}$ is empty or not. If the intersection is empty, $\rho$ is inseparable, otherwise $\rho$ is separable.

The steps from (i) to (iii) are easy to follow, but step (iv) is very difficult to operate at first sight. It is natural to ask whether step (iv) can be done or not and whether the method is practical . To answer this question and illustrate the method, we give an example of a bound entangled state.

A state $\rho$ of a $2 \times 4$ system [6] is

$$
\rho=\frac{1}{8}\left[\begin{array}{llllllll}
1 & 0 & 0 & 0 & 0 & 1 & 0 & 0 \\
0 & 1 & 0 & 0 & 0 & 0 & 1 & 0 \\
0 & 0 & 1 & 0 & 0 & 0 & 0 & 1 \\
0 & 0 & 0 & 1 & 0 & 0 & 0 & 0 \\
0 & 0 & 0 & 0 & 1 & 0 & 0 & 0 \\
1 & 0 & 0 & 0 & 0 & 1 & 0 & 0 \\
0 & 1 & 0 & 0 & 0 & 0 & 1 & 0 \\
0 & 0 & 1 & 0 & 0 & 0 & 0 & 1
\end{array}\right] .
$$

$\rho$ has five nonzero eigenvalues $t_{i}(i=1,2, \cdots, 5)$,

$$
\begin{aligned}
& t_{1}=t_{2}=\frac{1}{8}, \\
& t_{3}=t_{4}=t_{5}=\frac{1}{4},
\end{aligned}
$$

The corresponding unnormalized eigenvectors $\left|x_{i}\right\rangle$ can be

$$
\begin{aligned}
& \left|x_{1}\right\rangle=\left(0,0,0, \sqrt{\frac{1}{8}}, 0,0,0,0\right),\left|x_{2}\right\rangle=\left(0,0,0,0, \sqrt{\frac{1}{8}}, 0,0,0\right), \\
& \left|x_{3}\right\rangle=\left(\sqrt{\frac{1}{8}}, 0,0,0,0, \sqrt{\frac{1}{8}}, 0,0\right),\left|x_{4}\right\rangle=\left(0, \sqrt{\frac{1}{8}}, 0,0,0,0, \sqrt{\frac{1}{8}}, 0\right), \\
& \left|x_{5}\right\rangle=\left(0,0, \sqrt{\frac{1}{8}}, 0,0,0,0, \sqrt{\frac{1}{8}}\right) .
\end{aligned}
$$

The three matrices $B^{r}(r=1,2,3)$ corresponding to the three parallel pairs are

$$
\begin{aligned}
& \left(A_{11}, A_{12}\right) \|\left(A_{21}, A_{22}\right) \rightarrow B_{1,6}^{1}=B_{6,1}^{1}=-1, B_{2,5}^{1}=B_{5,2}^{1}=1, \\
& \left(A_{11}, A_{13}\right) \|\left(A_{21}, A_{23}\right) \rightarrow B_{1,7}^{2}=B_{7,1}^{2}=-1, B_{3,5}^{2}=B_{5,3}^{2}=1, \\
& \left(A_{11}, A_{14}\right) \|\left(A_{21}, A_{24}\right) \rightarrow B_{1,8}^{3}=B_{8,1}^{3}=-1, B_{4,5}^{3}=B_{5,4}^{3}=1 .
\end{aligned}
$$


Then the symmetric matrices $\tau_{i j}^{r}=\left\langle x_{i} \mid \widetilde{x}_{j}^{r}\right\rangle=\left\langle x_{i}\left|B^{r}\right| x_{j}^{*}\right\rangle \quad(i, j=1,2, \cdots, 5, r=1,2,3)$ are

$$
\tau^{1}=\left[\begin{array}{ccccc}
0 & 0 & 0 & 0 & 0 \\
0 & 0 & 0 & \frac{1}{8} & 0 \\
0 & 0 & -\frac{1}{4} & 0 & 0 \\
0 & \frac{1}{8} & 0 & 0 & 0 \\
0 & 0 & 0 & 0 & 0
\end{array}\right], \tau^{2}=\left[\begin{array}{ccccc}
0 & 0 & 0 & 0 & 0 \\
0 & 0 & 0 & 0 & \frac{1}{8} \\
0 & 0 & 0 & -\frac{1}{8} & 0 \\
0 & 0 & -\frac{1}{8} & 0 & 0 \\
0 & \frac{1}{8} & 0 & 0 & 0
\end{array}\right], \tau^{3}=\left[\begin{array}{ccccc}
0 & \frac{1}{8} & 0 & 0 & 0 \\
\frac{1}{8} & 0 & 0 & 0 & 0 \\
0 & 0 & 0 & 0 & -\frac{1}{8} \\
0 & 0 & 0 & 0 & 0 \\
0 & 0 & -\frac{1}{8} & 0 & 0
\end{array}\right]
$$

It is easy to get that $a^{1}=0, a^{2}<0, a^{3}<0$, so we must turn to step (iv). According to the theorem, there are surely three sets of transformation matrices $u_{k \times 5}$ each of which can result in the decomposition $\left|z_{i}\right\rangle$ of $\rho, \rho=\sum_{i=1}^{k}\left|z_{i}\right\rangle\left\langle z_{i}\right|$ with the corresponding pair being parallel. The $u_{k \times 5}$ can be written as

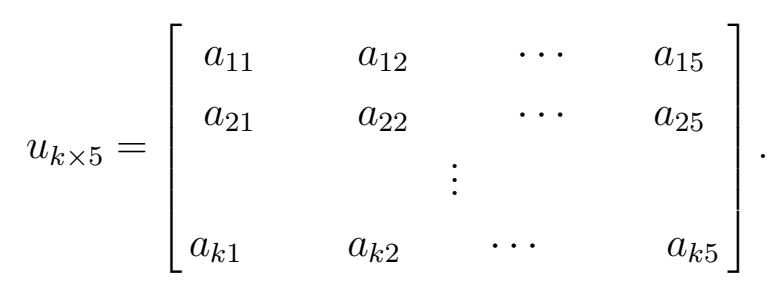

Each column in $u_{k \times 5}$ is an orthonormal vector. If three sets of $u_{k \times 5}$ have intersection, the state $\rho$ is separable. This condition is equivalent to the existence of a set $a_{11}, \cdots, a_{15}, \cdots, a_{k 1, \cdots,} a_{k 5}$ satisfying the following equations

$$
\left\{\begin{array}{l}
\left|z_{i}\right\rangle=a_{i 1}\left|x_{1}\right\rangle+a_{i 2}\left|x_{2}\right\rangle+\cdots+a_{i 5}\left|x_{5}\right\rangle \\
\left\langle z_{i}\left|B^{1}\right| z_{i}^{*}\right\rangle=0 \\
\left\langle z_{i}\left|B^{2}\right| z_{i}^{*}\right\rangle=0 \\
\left\langle z_{i}\left|B^{3}\right| z_{i}^{*}\right\rangle=0
\end{array} \quad(i=1,2, \cdots, k)\right.
$$

From Eqs.(21) and (23), one can get that

$$
\left\{\begin{array}{c}
a_{i 2}^{*} a_{i 4}^{*}=a_{i 3}^{* 2}, \\
a_{i 2}^{*} a_{i 5}^{*}=a_{i 3}^{*} a_{i 4}^{*}, \\
a_{i 1}^{*} a_{i 2}^{*}=a_{i 3}^{*} a_{i 5}^{*}
\end{array}\right.
$$

The complex number $a_{i j}^{*}$ can be expressed as $a_{i j}^{*}=b_{i j} e^{i \theta_{i j}}$ where $b_{i j}$ is a real and positive number. From the first equation of Eq.(24), one can get that $\sum_{i=1}^{k} b_{i 2} b_{i 4}=\sum_{i=1}^{k} b_{i 3}^{2}=1$. Because $\sum_{i=1}^{k} b_{i j}^{2}=1$, the equality $b_{i 2}=b_{i 3}=b_{i 4}$ can be obtained. Then Eq. (24) results in

$$
\left\{\begin{array}{c}
b_{i 1}=b_{i 2}=b_{i 3}=b_{i 4}=b_{i 5}=b_{i} \\
\theta_{i 3}=\frac{\theta_{i 1}+3 \theta_{i 2}}{4}, \theta_{i 4}=\frac{\theta_{i 1}+\theta_{i 2}}{2}, \theta_{i 5}=\frac{3 \theta_{i 1}+\theta_{i 2}}{4} .
\end{array}\right.
$$

Because each column in Eq.(22) is orthonormal, one can obtain that 


$$
\left\{\begin{array}{c}
\sum_{i=1}^{k} b_{i}^{2} e^{i\left(\theta_{i 1}-\theta_{i 2}\right)}=0 \\
\sum_{i=1}^{k} b_{i}^{2} e^{i\left(\theta_{i 1}-\theta_{i 3}\right)}=\sum_{i=1}^{k} b_{i}^{2} e^{i \frac{3\left(\theta_{i 1}-\theta_{i 2}\right)}{4}}=0 \\
\sum_{i=1}^{k} b_{i}^{2} e^{i\left(\theta_{i 1}-\theta_{i 4}\right)}=\sum_{i=1}^{k} b_{i}^{2} e^{i \frac{\theta_{i 1}-\theta_{i 2}}{2}}=0
\end{array}\right.
$$

It is easy to find that Eq. (26) has no solution since $\sum_{i=1}^{k} b_{i}^{2} e^{i\left(\theta_{i 1}-\theta_{i 2}\right)}=0, \sum_{i=1}^{k} b_{i}^{2} e^{i \frac{3\left(\theta_{i 1}-\theta_{i 2}\right)}{4}}=$ 0 , and $\sum_{i=1}^{k} b_{i}^{2} e^{i \frac{\theta_{i 1}-\theta_{i 2}}{2}}=0$ do not hold true simultaneously.. Therefore the intersection $u_{k \times 5}$ of three sets of transformation matrix is empty and $\rho$ in Eq.(17) is inseparable.

From the above example, one can see that for any mixed state the problem of finding the intersection of our sets of transformation matrices comes down to the problem of finding the solution of a set of quadratic equations with the condition of the solution being orthonormal vectors. The condition of orthonormality that makes the quadratic equations more easily solved. Especially, if there are not many nonzero elements of the symmetry matrix $\tau^{r}$, it is very easy to solve the set of quadratic equations. In addition, the form of these equations is dependent on the choice of eigenvectors of $\rho$, which implies that one may simplify the form of these equations with appropriate eigenvectors of $\rho$.

In summary, we have presented a new necessary and sufficient condition for separability of state of any system. According to the above theorem, $(m-1) \times(n-1)$ conditions should be tested in order to judge whether a state is separable or not. Though in mathematical form this method is more complex than the one provided in [9], it is practical in judging the separability of a state. Meanwhile, our theorem provides a method to find the disentangled decomposition of a separable state. It is also worth mentioning that for $2 \times 2$ systems, the condition that the partial transposition(PT) is positive and the condition that each $a^{1} \leq 0$ in our method are equivalent. This suggests that there may be an essential connection between the PT of $\rho$ and $a^{1}$.

\section{ACKNOWLEDGMENTS}

We thank William K. Wootters and Guangcan Guo for their encouragement. This work was supported in part by the National Natural Science Foundation of China.

\section{APPENDIX}

Considering an $m \times n$ system, a state $\rho$ of this system, which has $l$ nonzero eigenvalues, has a decomposition, $\rho=\sum_{i}\left|y_{i}\right\rangle\left\langle y_{i}\right|$, which satisfies $\left\langle y_{i} \mid \widetilde{y}_{j}^{r}\right\rangle=\lambda_{i} \delta_{i j}$, where $\left|\widetilde{y}_{i}^{r}\right\rangle=B^{r}\left|y_{i}^{*}\right\rangle$ and $B^{r}$ is a transformation matrix corresponding to a parallel pair $(r=1,2, \cdots,(m-$ $1)(n-1))$. If $\lambda_{1} \leq \sum_{i=2}^{l^{\prime}} \lambda_{i}\left(l^{\prime}\right.$ is the number of nonzero eigenvalues of $\left.\tau^{r}\left(\tau^{r}\right)^{*}\right)$, one can find a decomposition of $\rho$,

$$
\left|z_{i}\right\rangle=\frac{1}{2 \sqrt{k}} \sum_{j=1}^{l} a_{i j} e^{i \theta_{j}}\left|y_{j}\right\rangle, \quad i=1,2, \cdots, 4 k, \quad k=1,2,3, \cdots
$$


where $4 k$ is greater than or equal to $l,\left|a_{i j}\right|=1$ and their signs are decided by Fig. 1 , $\left|y_{l^{\prime}+1}\right\rangle,\left|y_{l^{\prime}+2}\right\rangle, \cdots,\left|y_{l}\right\rangle$ being zero vectors. Phase factors can be found to make $\sum_{j} e^{2 i \theta_{j}} \lambda_{j}=$ 0 [11], which guarantees $\left\langle z_{i} \mid \widetilde{z}_{i}^{r}\right\rangle=0$. Obviously, the matrices $\left[a_{i j}\right]$ is a $4 k \times l$ matrix.

From Fig. 1, one can find that

$$
\begin{aligned}
& a_{4 \times 4}=\left[\begin{array}{cccc}
+1 & +1 & +1 & +1 \\
+1 & +1 & -1 & -1 \\
+1 & -1 & +1 & -1 \\
+1 & -1 & -1 & +1
\end{array}\right] \text {. }
\end{aligned}
$$

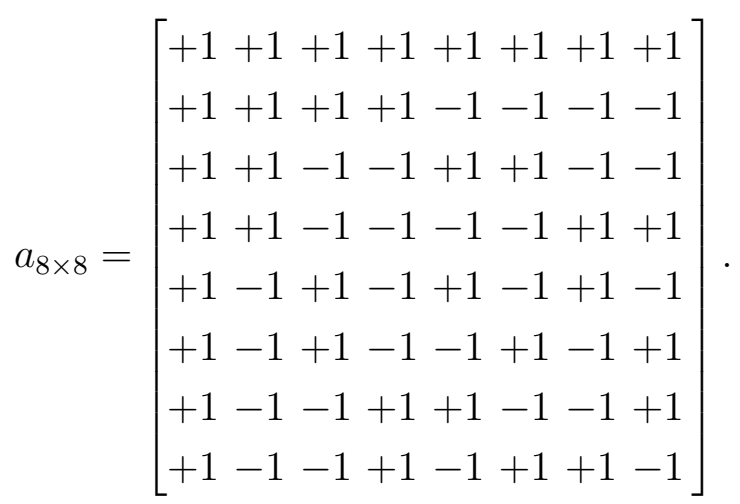

According to the symmetry, one can find the higher-dimension matrix $a_{4 k \times 4 k}$ easily. If the number $l$ of nonzero eigenvalues of $\rho$ is less than $4 k$, one may take the first $l$ columns of $a_{4 k \times 4 k}$. For example,

$$
a_{8 \times 5}=\left[\begin{array}{lllll}
+1 & +1 & +1 & +1 & +1 \\
+1 & +1 & +1 & +1 & -1 \\
+1 & +1 & -1 & -1 & +1 \\
+1 & +1 & -1 & -1 & -1 \\
+1 & -1 & +1 & -1 & +1 \\
+1 & -1 & +1 & -1 & -1 \\
+1 & -1 & -1 & +1 & +1 \\
+1 & -1 & -1 & +1 & -1
\end{array}\right] .
$$




\section{REFERENCES}

[1] J.S. Bell, Physics 1, 195(1964).

[2] D.P. DiVincenzo, Science 270, 255(1995).

[3] C.A. Fuchs, N. Gisin, R.B. Griffiths, C.S. Niu, and A. Peres, Phys. Rev. A 56,1163(1997).

[4] C. H. Bennett, H. J. Bernstein, S. Popescu and B. Schumacher, Phys. Rev. A 53 2046(1996) .

[5] C. H. Bennett, D. P. Divincenzo, J. A. Smolin, and W. K. Wootters, Phys. Rev. A 54 $3824(1996)$.

[6] P. Horodecki, Phys. Lett. A 232, 333(1997).

[7] A. Peres, Phys. Rev. A 54, 2685(1996).

[8] A. Peres, Phys. Rev. Lett. 77, 1413(1996).

[9] M. Horodecki, P. Horodecki, and R. Horodecki, Phys. Lett. A 223, 1(1996).

[10] W. Dür, J.I.. Cirac, and R. Tarrach, Phys. Rev. Lett. 83, 3562(1999).

[11] W. K.Wootters, Phys. Rev. Lett. 80, 2245(1998).

[12] S. Hill and W. K.Wootters, Phys. Rev. Lett. 78, 5022(1997). 


\section{Figure Captions}

Fig. 1. The diagram showing the sign of the oefficient $a_{k j}$ in Eq. (16). 


\section{FIGURES}

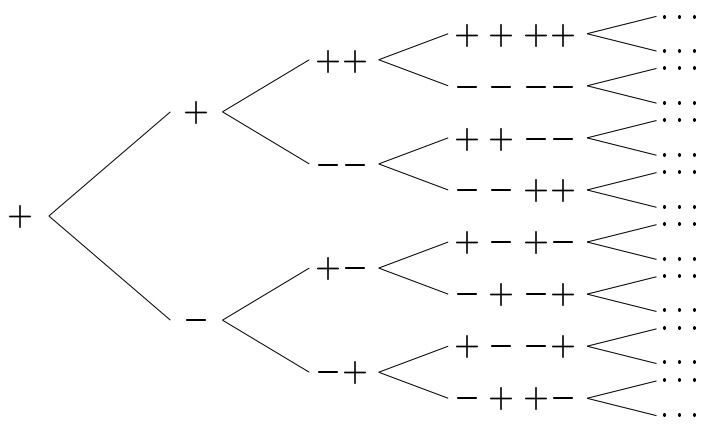

Fig 1 